\title{
ON A THEOREM OF MARCINKIEWICZ AND ZYGMUND
}

\author{
BY \\ A. P. CALDERÓN( ${ }^{(1)}$
}

The purpose of the present paper is to prove the following result:

Let $F(P), P=\left(x_{1}, x_{2}, \cdots, x_{n}\right)$, be a function harmonic for $x_{n}>0$ and such that for every point $Q$ of a set $E$ of positive measure on the hyperplane $x_{n}=0$ there exists a region contained in $x_{n}>0$ limited by a cone with vertex at $Q$ and a hyperplane $x_{n}=$ const. where the function is bounded. Then except for a set of measure zero, the integral

$$
\int \frac{1}{x_{n}^{n-2}} \operatorname{grad}^{2} F d \omega,
$$

extended over any region limited by a cone with vertex at $Q \in E$, a hyperplane $x_{n}=$ const., and contained in $x_{n}>0$, is finite.

The case $n=2$ of the theorem was established by Marcinkiewicz and Zygmund $[1]\left({ }^{2}\right)$ using methods of conformal mapping; here we give a proof of it which does not depend on analytic functions and which can be extended without change to functions of any number of variables. It is interesting to remark that the integral (6) below, which appears in the argument in a natural way, was introduced by Marcinkiewicz for functions of a single variable and used by him to prove difficult results of the theory of Fourier series. The same integral for functions of several variables was also considered by $Z$ ygmund in an unpublished paper.

In the case of two variables the converse of the statement above holds, as shown by Spencer [2]. Its validity in the general case remains an open problem.

For the sake of simplicity we shall confine our attention to functions of three variables $F(x, y, z)$ harmonic for $z>0$, the argument applying without change to the general case.

First we may remark that it suffices to show that whenever the function satisfies the boundedness condition for a set $E$ of positive measure, there exists a subset $E^{\prime}$ of $E$, also of positive measure, for which the integral (1) is finite. In fact, the theorem follows by an obvious argument.

Another less obvious remark is the following:

Let us denote by $\Gamma(Q, \alpha)$ the intersection of the cone parallel to $x^{2}+y^{2}<\alpha^{2} z^{2}$ with vertex at $Q$ and the layer $0<z<1$. Then we may assume

Presented to the Society, September 2, 1949; received by the editors December 13, 1948, and, in revised form, July 20, 1949.

(1) Fellow of the Rockefeller Foundation.

(2) Numbers in brackets refer to the references cited at the end of the paper. 
that for every point $Q$ of $E, F(P)$ is bounded not only in some conical region with vertex at $Q$, but is uniformly bounded in all $\Gamma(Q, \alpha), Q \in E$, for every fixed $\alpha$. Under the assumptions of our theorem this is indeed always true for some subset of positive measure of $E$.

To show this, consider a sequence of cones $\Gamma^{(n)}$ such that every given cone contains at least one parallel to some $\Gamma^{(n)}$; such a sequence clearly exists. Let now $E_{n}^{m}$ be the set of points $Q$ of $z=0$, such that $|F(P)|<m$ for $0<z<1$ when $P$ belongs to a cone parallel to $\Gamma^{(n)}$ with vertex at $Q$. Since $F$ is continuous in $z>0$ the sets $E_{n}^{m}$ are measurable and every point of $E$ belongs to some $E_{n}^{m}$, that is, $E \subset \cup E_{n}^{m}$. Let now $Q$ be a point of density of $E_{n}^{m}$; consider $\Gamma(Q, \alpha)$ and a point $P$ of it; draw with vertex at $P$ a cone parallel to $\Gamma^{(n)}$ directed towards the plane $z=0$ and intersecting it in the ellipse $\Sigma$; as $P$ tends to $Q$ remaining inside $\Gamma(Q, \alpha)$ the average density of $E_{n}^{m}$ in $\Sigma$ tends to 1 , and so when the coordinate $z$ of $P$ is small enough $\Sigma$ contains a point $Q^{\prime}$ of $E_{n}^{m}$. But this implies that $P$ is contained in the cone parallel to $\Gamma^{(n)}$ with vertex at $Q^{\prime}$, and hence that $|F(P)|<m$. So, for $z$ small enough, we have $|F(P)|<m$ in $\Gamma(Q, \alpha)$; and since $F$ is continuous in $z>0$, it is bounded in $\Gamma(Q, \alpha)$.

Now, since $E \subset \cup E_{n}^{m}$, almost every point of $E$ is a density point of some $E_{n}^{m}$, and so $F(P)$ is bounded in $\Gamma(Q, \alpha)$ for almost all $Q \in E$ and every $\alpha$. Finally let $E_{n m}$ be the set of points $Q$ such that $|F(P)|<m$ in $\Gamma(Q, n)$; clearly for every fixed $n$ we have $E_{n m} \subset E_{n, m+1}$ and, except for a set of measure zero, $E \subset \lim _{m \rightarrow \infty} E_{n m}$; hence for that $n$ we may find an $m=\phi(n)$ such that

$$
\left|E-E_{n, \phi(n)}\right| \leqq \frac{1}{2^{n+1}}|E|
$$

and putting $E^{\prime}=\bigcap_{1}^{\infty} E_{n, \phi(n)}$

$$
\left|E^{\prime}\right| \geqq|E|-\sum_{1}^{\infty}\left|E-E_{n, \phi(n)}\right| \geqq \frac{1}{2}|E| \text {. }
$$

Now at every point $Q$ of $E^{\prime}$ we have $|F(P)|<\phi(n)$ in $\Gamma(Q, n)$; that is, for every $n, F$ is uniformly bounded in all $\Gamma(Q, n), Q \in E^{\prime}$, as we wanted to show.

Hence from now on we are allowed to assume that $F(P)$ is uniformly bounded in all $\Gamma(Q, \alpha), Q \in E$, for every fixed $\alpha$.

In order to avoid later consideration of more sets, we shall assume in addition that $E$ is closed and contained in $x^{2}+y^{2} \leqq 1$. This clearly does not affect the validity of the argument.

We now pass to the proof of the theorem. Let $\gamma(P, Q)$ be the characteristic function of the set of points $P \Gamma(Q, \alpha)$. We have

$$
\int_{\Gamma(Q, \alpha)} \frac{1}{z} \operatorname{grad}^{2} F d v=\int \frac{1}{z} \gamma(P, Q) \operatorname{grad}^{2} F d v
$$

where the integral without subscript is extended over the whole space. In- 
tegrating this equality with respect to $Q$ over a set $E^{\prime}$ of $z=0$ we get

$$
\int_{E^{\prime}} d \sigma \int_{\Gamma_{(Q, \alpha)}} \frac{1}{z} \operatorname{grad}^{2} F d v=\int \frac{1}{z} \operatorname{grad}^{2} F \int_{E^{\prime}} \gamma(P, Q) d \sigma .
$$

Let us denote by $\Delta\left(E^{\prime}, \alpha\right)$ the union of all regions $\Gamma(Q, \alpha), Q \in E^{\prime}$. If $P$ is not contained in $\Delta\left(E^{\prime}, \alpha\right)$, no $\Gamma(Q, \alpha), Q \in E^{\prime}$, contains $P$ and so $\gamma(P, Q)=0$ for $Q \in E^{\prime}$, and

$$
\int_{E^{\prime}} \gamma(P, Q) d \sigma=0
$$

for that $P$. On the other hand, $\gamma(P, Q)$ vanishes if the distance of $Q$ from the projection $P^{\prime}$ of $P$ on $z=0$ exceeds $\alpha z, z$ being the coordinate of $P$. Hence the integral of $\gamma(P, Q)$ over $E^{\prime}$ never exceeds the area of the circle with center at $P^{\prime}$ and radius $\alpha z$; that is,

$$
\int_{E^{\prime}} \gamma(P, Q) d \sigma \leqq \pi \alpha^{2} z^{2}
$$

Then substituting above we get

$$
\int_{E^{\prime}} d \sigma \int_{\Gamma(Q, \alpha)} \frac{1}{z} \operatorname{grad}^{2} F d v \leqq \pi \alpha^{2} \int_{\Delta\left(E^{\prime}, \alpha\right)} z \operatorname{grad}^{2} F d v
$$

This shows that the finiteness of the integral at the right over $\Delta\left(E^{\prime}, \alpha\right)$ implies the finiteness of (2) for almost all $Q \in E^{\prime}$. Thus, our problem reduces to show that for every $\alpha$ there exists a set $E^{\prime}$ such that the right-hand side above is finite.

In order to achieve this goal, for every $\alpha>0$ we shall construct a function $G(P)$ with the following properties:

(a) $G(P)$ is harmonic for $z>0$.

(b) $G(P) \geqq z$ in $\Delta\left(E^{\prime}, \alpha\right)$, where $E^{\prime}$ is a subset of positive measure of $E$, which depends on $\alpha$.

(c) Let $D$ denote the set of points $P$ situated in the cylinder $x^{2}+y^{2} \leqq(1+\alpha)^{2}$ and in the layer $0<z<1$, such that $G(P) \geqq 0$. Then $D$ is contained in $\Delta(E, \beta)$ for some $\beta>\alpha$.

Since $G(P) \geqq z$ in $\Delta\left(E^{\prime}, \alpha\right)$, and $\Delta\left(E^{\prime}, \alpha\right)$ is contained in the cylinder $x^{2}+y^{2} \leqq(1+\alpha)^{2}$, we have $\Delta\left(E^{\prime}, \alpha\right) \subset D \subset \Delta(E, \beta)$ and

$$
\int_{D} G(P) \operatorname{grad}^{2} F d v \geqq \int_{د\left(E^{\prime}, \alpha\right)} z \operatorname{grad}^{2} F d v .
$$

Now, owing to the properties of $G(P)$ it will not be difficult to show that the left-hand side is finite.

Let us denote by $B$ the boundary of $D$, by $B_{1}$ the part of it at which 
$G(P)=0$, and by $B_{2}$ the remainder of $B$. Assuming for a while that the boundary of $D$ is sufficiently regular and applying Green's formula, we have

$$
\int_{B}\left(G \frac{\partial F^{2}}{\partial n}-F^{2} \frac{\partial G}{\partial n}\right) d \sigma=2 \int_{D} G \operatorname{grad}^{2} F d v
$$

Now $G(P)$ vanishes on $B_{1}$ and $G \geqq 0$ in $D$, hence $\partial G / \partial n \leqq 0$ on $B_{1}$. On the other hand

$$
\int_{B} \frac{\partial G}{\partial n} d \sigma=\int_{B_{1}} \frac{\partial G}{\partial n} d \sigma+\int_{B_{2}} \frac{\partial G}{\partial n} d \sigma=\int_{D} \nabla G d v=0 .
$$

But the points of $B_{2}$ are situated either on the cylinder $x^{2}+y^{2}=(1+\alpha)^{2}$ or on the plane $z=1$, and always in $\Delta(E, \beta)$. Since $E$ is contained in the circle $x^{2}+y^{2}=1$, it is easily seen from the definition of $\Delta$ that the intersection of $\Delta(E, \beta)$ with $x^{2}+y^{2}=(1+\alpha)^{2}$ is at a positive distance from $z=0$. Hence $B_{2}$ is also at a positive distance from $z=0$ and so $\partial G / \partial n$ is continuous and bounded there, and the integral over $B_{2}$ is finite. Now, we had $\partial G / \partial n \leqq 0$ on $B_{1}$; on the other hand $B_{1} \subset D \subset \Delta(E, \beta)$, and because $F$ is uniformly bounded in all $\Gamma(Q, \beta)$ for $Q \in E$, it is bounded in $\Delta(E, \beta)$, and therefore also in $B_{1}$. Then

$$
0 \geqq \int_{B_{1}} F^{2} \frac{\partial G}{\partial n} d \sigma>-\infty .
$$

Substituting above and recalling that $G=0$ on $B_{1}$ we get

$$
\int_{B_{2}}\left(G \frac{\partial F^{2}}{\partial n}-F^{2} \frac{\partial G}{\partial n}\right) d \sigma-\int_{B_{1}} F^{2} \frac{\partial G}{\partial n} d \sigma=2 \int G \operatorname{grad}^{2} F d v .
$$

Again, since $B_{2}$ is at a positive distance from $z=0$, all functions appearing in the integral over $B_{2}$ are continuous and bounded, and so the left-hand side is finite, and this would establish our theorem. In order to avoid troubles with the boundary of $D$, we can apply Green's formula to the functions $G(P)-\epsilon$ and $F^{2}$ over the domain $D_{\epsilon}$ of points contained in $0<z<1$ and $x^{2}+y^{2} \leqq(1+\alpha)^{2}$ where $G(P)-\epsilon \geqq 0$. As we shall see from the construction of $G, D_{\epsilon}$ is entirely contained in $z>0$ and its boundary is quite regular. Then, arguing in the same way as above, we would obtain

$$
\int_{D_{\epsilon}}(G-\epsilon) \operatorname{grad}^{2} F d v \leqq K,
$$

$K$ being a constant independent of $\epsilon$. Let us repeat the argument briefly. Denoting by $B_{\epsilon}$ the boundary of $D_{\epsilon}$, by $B_{1 \epsilon}$ the part of $B_{\epsilon}$ at which $G(P)-\epsilon=0$ and by $B_{2 \epsilon}$ the remainder, and applying Green's formula to $G-\epsilon$, we have

$$
\int_{B_{1 \epsilon}} \frac{\partial(G-\epsilon)}{\partial n} d \sigma+\int_{B_{2 \epsilon}} \frac{\partial(G-\epsilon)}{\partial n} d \sigma=\int_{D_{\epsilon}} \nabla(G-\epsilon) d v=0 .
$$


Again, the integral over $B_{2 \epsilon}$ is finite and bounded as $\epsilon \rightarrow 0$, as is easily seen by the same reasons as given above. Also $\partial(G-\epsilon) / \partial n \leqq 0$ on $B_{2 \epsilon}$ and $F$ is uniformly bounded in all $B_{1 \epsilon}$; therefore

$$
0 \geqq \int_{B_{1 \epsilon}} F^{2} \frac{\partial(G-\epsilon)}{\partial n} d \sigma>-k
$$

for some fixed $k>0$ independent of $\epsilon$. Applying Green's formula again we get

$$
\int_{B_{\epsilon}}\left[(G-\epsilon) \frac{\partial F^{2}}{\partial n}-F^{2} \frac{\partial(G-\epsilon)}{\partial n}\right] d \sigma=2 \int_{D_{\epsilon}}(G-\epsilon) \operatorname{grad}^{2} F d v
$$

But since $G-\epsilon=0$ on $B_{1 \epsilon}$, the right-hand side reduces to

$$
\int_{B_{2 \epsilon}}\left[(G-\epsilon) \frac{\partial F^{2}}{\partial n}-F^{2} \frac{\partial(G-\epsilon)}{\partial n}\right] d \sigma-\int_{B_{1 \epsilon}} F^{2} \frac{\partial(G-\epsilon)}{\partial n} d \sigma .
$$

The second integral remains bounded as we have shown; the first is also bounded, as before; hence we get (3).

Making $\epsilon$ tend to zero in (3) we obtain finally

$$
\int_{D} G \operatorname{grad}^{2} F d v \leqq K
$$

as we wanted to show.

It remains only to construct the function $G(P)$ and to prove that it fulfills the required properties.

Let us denote by $\delta(Q)$ the distance of the point $Q$ of $z=0$ from the set $E$. We define

$$
G(P)=k z-\int \frac{z \delta(Q)}{|P-Q|^{3}} d \sigma,
$$

the integral being extended over the circle $x^{2}+y^{2} \leqq(3+2 \alpha)^{2},|P-Q|$ denoting the distance from $P$ to $Q$, and $k$ being a positive constant depending on $\alpha$ which we shall fix later.

First, we shall show that

$$
\int \frac{\delta(Q)}{|P-Q|^{3}} d \sigma
$$

is bounded in some $\Delta\left(E^{\prime}, \alpha\right), E^{\prime}$ being a positive subset of $E$, in such a way that choosing $k$ large enough, we shall have $G \geqq z$ in $\Delta\left(E^{\prime}, \alpha\right)$.

In order to prove this consider the integral

$$
\int \frac{\delta(Q)}{\left|Q^{\prime}-Q\right|^{3}} d \sigma
$$


extended over $x^{2}+y^{2} \leqq(3+2 \alpha)^{2}, Q^{\prime}$ being a point of $z=0$. Since $\delta(Q)$ vanishes in $E$, denoting by $\bar{E}$ the set complementary to $E$ in $x^{2}+y^{2} \leqq(3+2 \alpha)^{2}$, we have

$$
\int \frac{\delta(Q)}{\left|Q^{\prime}-Q\right|^{3}} d \sigma=\int_{E} \frac{\delta(Q)}{\left|Q^{\prime}-Q\right|^{3}} d \sigma .
$$

Integrating with respect to $Q^{\prime}$ over $E$ and inverting the order of integration, we obtain

$$
\int_{E} d \sigma^{\prime} \int_{E} \frac{\delta(Q)}{\left|Q^{\prime}-Q\right|^{3}} d \sigma=\int_{E} \delta(Q) d \sigma \int_{E} \frac{d \sigma^{\prime}}{\left|Q^{\prime}-Q\right|^{3}} .
$$

But since $E$ is closed, for every $Q$ not contained in $E, \delta(Q)$ is positive, and the inner integral at the right does not exceed

$$
\int_{\delta(Q)}^{2(2+\alpha)} \frac{2 \pi r d r}{r^{3}}=\frac{2 \pi}{\delta(Q)}-\frac{\pi}{(2+\alpha)} .
$$

Substituting this expression above it follows immediately that the left-hand member is finite and that, hence, (6) is finite for almost every $Q^{\prime}$ of $E$.

Next, let $Q^{\prime}$ be a point of $E$, and $P$ a point of $\Gamma\left(Q^{\prime}, \alpha\right)$. Then

$$
\int \frac{\delta(Q)}{|P-Q|^{3}} d \sigma=\int \frac{\delta(Q)}{\left|Q^{\prime}-Q\right|^{3}} \cdot \frac{\left|Q^{\prime}-Q\right|^{3}}{|P-Q|^{3}} d \sigma
$$

but since $Q$ belongs to $z=0$ and $P$ is contained in $\Gamma\left(Q^{\prime}, \alpha\right)$, elementary computations show that

$$
\frac{\left|Q^{\prime}-Q\right|^{3}}{|P-Q|^{3}} \leqq\left(1+\alpha^{2}\right)^{3 / 2}
$$

whence, replacing in the foregoing expression, we obtain

$$
\int \frac{\delta(Q)}{|P-Q|^{3}} d \sigma \leqq\left(1+\alpha^{2}\right)^{3 / 2} \int \frac{\delta(Q)}{\left|Q^{\prime}-Q\right|^{3}} d \sigma .
$$

Now, the right-hand side is finite for almost every $Q^{\prime}$ of $E$ and hence it is bounded for $Q^{\prime}$ belonging to a suitable subset $E^{\prime}$ of positive measure of $E$; that is, the integral(5) is bounded for $P$ belonging to $\Delta\left(E^{\prime}, \alpha\right)$, as we wanted to show. But

$$
G(P)=z\left[k-\int \frac{\delta(Q)}{|P-Q|^{3}} d \sigma\right],
$$

therefore, choosing $k$ large enough, property (b) follows.

Finally, to verify (c), take any $\beta>\alpha$ and consider $\Delta(E, \beta)$. If $P$ is a point of the cylinder $x^{2}+y^{2} \leqq(1+\alpha)^{2}$ not contained in $\Delta(E, \beta)$, and $P^{\prime}$ is its projection on $z=0$, the distance from $P^{\prime}$ to $E$ is not less than $\beta z, z$ being the coordi- 
nate of $P$. Hence, introducing polar coordinates in (4), we have

$$
G(P)=k z-\int \frac{z \delta(Q)}{|P-Q|^{3}} d \sigma \leqq k z-2 \pi \int_{0}^{\beta z} \frac{z(\beta z-r) r}{\left(z^{2}+r^{2}\right)^{3 / 2}} d r
$$

and

$$
G(P) \leqq k z-2 \pi z \int_{0}^{\beta} \frac{(\beta-s) s d s}{\left(1+s^{2}\right)^{3 / 2}},
$$

and if $\beta$ is taken sufficiently large, the desired result follows.

It remains to verify the properties of $D_{\epsilon}$ required to allow the application of Green's formula.

First of all, it is easily seen from (4) that $G(P)$ has a nonpositive upper limit when $P$ tends to a point of $z=0$. Hence every point $P$ at which $G(P)$ $\geqq \epsilon>0$ is contained in $z>0$, and the closed domain $D_{\epsilon}$ is also contained in $z>0$. On the other hand

$$
G(P)=z\left[k-\int \frac{\delta(Q)}{|P-Q|^{3}} d \sigma\right] ;
$$

the integral inside is easily seen to be a nonincreasing function of $z$, and since $G(P)>0$ on $G(P)=\epsilon$, we have

$$
k-\int \frac{\delta(Q)}{|P-Q|^{3}} d \sigma>0
$$

and therefore $\partial G / \partial z>0$ there. Hence the surface $G(P)-\epsilon=0$ has everywhere a tangent plane which varies continuously along it. Now the boundary of $D_{\epsilon}$ consists of three parts: one, a portion of the surface where $G(P)-\epsilon=0$, a second part contained in $z=1$, and perhaps a third part contained in $x^{2}+y^{2}$ $=(1+\alpha)^{2}$. This shows that the application of Green's formula is correct.

\section{REFERENCES}

1. J. Marcinkiewicz and A. Zygmund, A theorem of Lusin, Duke Math. J. vol. 4 (1938) pp. 473-485.

2. D. C. Spencer, A function theoretic identity, Amer. J. Math. vol. 65 (1943) pp. 147-160.

University of Chicago,

Chicago, ILl. 